\title{
The Effects of Training on Music Perception and Appreciation for Cochlear Implant Recipients
}

\author{
Valerie Looi, ${ }^{1}$ Yuhan Wong, ${ }^{2}$ and Jenny H. Y. Loo ${ }^{2}$ \\ ${ }^{1}$ SCIC Cochlear Implant Program, RIDBC Service, The Australian Hearing Hub, Sydney, NSW 2109, Australia \\ ${ }^{2}$ Yong Loo Lin School of Medicine, National University of Singapore, Singapore 119077 \\ Correspondence should be addressed to Valerie Looi; valerie.looi@scic.org.au
}

Received 25 August 2015; Accepted 8 December 2015

Academic Editor: Giuseppe Mercante

Copyright (c) 2016 Valerie Looi et al. This is an open access article distributed under the Creative Commons Attribution License, which permits unrestricted use, distribution, and reproduction in any medium, provided the original work is properly cited.

\begin{abstract}
The aim of this study was to compare the effectiveness of a music appreciation training program (MATP) to that of focused music listening (FML) for improving music and/or speech in noise perception for postlingually deafened cochlear implant (CI) recipients. It was hypothesized that the MATP would show greater improvements than FML. Ten CI recipients were randomly divided into two groups: one undertaking the MATP and the other undertaking FML. Participants completed four 30-minute sessions per week for 8 weeks, with tests of music and speech-in-noise perception being administered four times per participant: before and after a control period, immediately after the intervention, and 4-8 weeks after intervention. There was a significant pre- to posttraining difference for the MATP group on the instrument identification test, as well as for half of the quality rating assessments. Although no statistically significant improvements were obtained for the FML group, there was a trend of higher scores postintervention for the instrument and ensemble identification tests, and compliance was substantially better than for the MATP group. While the results showed that only the music training significantly improved music perception, the potential of FML to benefit some CI recipients for some tasks was also observed.
\end{abstract}

\section{Introduction}

Expeditious progress in the field of cochlear implants (CI) has resulted in greater expectations from recipients and potential recipients. They hope to achieve more than just speech understanding in quiet, and research accordingly has expanded towards improving the perception of more complex stimuli such as speech-in-noise and music. The perception of music, in particular, has become increasingly important for recipients, families, researchers, and manufacturers. Music is a pervasive acoustic stimulus that, apart from being a form of entertainment, has cultural and social significance as well as implications on psychological wellbeing [1]. Listening to music has been rated as one of the most popular leisure activities and something important in people's lives [2].

Music has been shown to serve many roles for people of all ages, from regulating moods to developing self-awareness, as well as promoting social interaction [2-4]. A study in Australia of adults aged 65 or older found that music was important to them for connecting with other people, for maintaining well-being, as a source of entertainment, and as an experience associated with meaning and emotions [5]. A recent review on the relationship between music, health, and well-being highlighted how musical activities are beneficial to psychological health and well-being [6].

It is well established that postlingually deafened adult CI recipients are poorer than normally hearing $(\mathrm{NH})$ listeners at perceptual music tasks and also rate music to sound less pleasant and enjoyable. These issues stem from a combination of factors including the CI technology itself, psychoacoustic limitations associated with severe to profound hearing loss, and individual patient factors. Comprehensive reviews of these issues as well as published results of CI music perception abilities are provided by [7-10].

In recent years, the body of research looking to address these challenges has been growing. As some of the issues impacting on CI music perception stem back to the poor perception of pitch through the implant and some of the technological limitations of the device, CI manufacturers 
have been working to improve their technology such as trialling new speech processing strategies. However, improvements in CI signal processing have not shown corresponding improvements in music perception. For example, Gfeller et al. [11] retrospectively analysed results for 209 CI recipients to look for factors which may predict music perception scores. Neither speech processing strategy nor device manufacturer was a significant predictor. Further, there is little difference in perceptual acuity scores or subjective quality ratings between earlier [12, 13] and more-recent [14] studies by Looi et al., in which the same test materials were used, but the CI technology has been upgraded.

An alternative approach to improving music perception and enjoyment in CI users is through music training. This has been shown in recent research studies to have the potential to benefit recipients, despite the limitations in the technology (see [9] for a review). Further, music training can be offered to all CI recipients irrespective of the device(s) they use or any subsequent technological updates. The underlying premise to training is neural plasticity and the brain's capacity to learn and adapt. Neuroplasticity refers to the changes in the central nervous system's neural pathways in response to changes in the environment. It occurs not only in infants and children but in adults as well. Auditory training, defined as a focused, systematic exposure to particular auditory stimuli intended to improve a listener's ability to perceive these stimuli, can help CI recipients to adapt to degraded signals and electrical stimulation [15]. Although auditory training has predominantly focused on speech stimuli, these principles are applicable to music perception. As incidental use of the $\mathrm{CI}$ and music exposure (i.e., time with the $\mathrm{CI}$ ) has not been shown to improve music listening, specific training and/or focused listening with music stimuli is required [8-13]. The only factors that have been consistently shown to correlate with music perception test scores are age (younger age associated with better scores), postimplant music listening (more listening associated with better scores), and the use of a contralateral hearing aid for recipients with aidable residual hearing [9, 11]. Looi et al. [9] provide a comprehensive review of published music training studies for CI recipients, outcomes, and factors that impact on outcomes.

Interestingly, in the field of auditory neuroscience, there is emerging evidence that music training may lead to plasticitydriven changes in the auditory system that extend beyond the areas of the brain most associated with music listening [16]. This is brought about by cross-domain plasticity, whereby experience or training in one domain (e.g., music) causes changes in neural processing in another domain (e.g., speech). An example of this in $\mathrm{NH}$ adults is research by Parbery-Clark et al. $[17,18]$ which demonstrated that musicians exhibited slightly better speech-in-noise scores than nonmusicians. Although these studies have yet to be undertaken in CI recipients, it does provide the foundation to speculate that music training for $\mathrm{CI}$ users may have the potential to improve not only their music perception and enjoyment but also other auditory processes like speech perception in noise and pitch-based prosody perception [19].

Besides music training, focused music listening has also been suggested to help improve music enjoyment in CI users, with some studies using these terms interchangeably [20-22]. However, there has yet to be a study comparing a specific music training program to focused music listening. While the former would provide more structure, feedback, and listening guidance for the user, the latter would enable greater flexibility and personalisation which may encourage better compliance. Hence, the aim of this study was to compare the effectiveness of a computer-based music training program with focused music listening for music perception, music appreciation (quality ratings), and speech perception in noise. It was hypothesized that CI users who undertook the computer-based music training would show greater improvements in each of these three areas than those who carried out focused music listening alone.

\section{Methods}

Ethical approval for this research was obtained from the Domain Specific Review Board of the National Healthcare Group, Singapore. All procedures were in accordance with this approval.

2.1. Participants. Ten CI recipients were recruited from the National University Hospital in Singapore. Participants had to be aged 13 years or older, have at least 6 months of CI experience, be fluent in English, have access to a computer for the duration of the study, and have no other cognitive, neurological, significant visual, or physical impediments that would impede their ability to undertake the training program or assessment tasks. In addition, participants were expected to have a stable CI program (i.e., MAP) over the period of the study. The training program did not require participants to have any knowledge of musical notation or technical musical terminology. Participants were randomly divided into two groups-one group to train with a computer-based Music Appreciation Training Program (MATP group) and one group to carry out focused music listening (FML group) over an eight-week period.

Participants in the MATP group were aged 13 to 31 years $(M=26 \mathrm{y})$, and those in the FML group were aged 15-47 years $(M=24 \mathrm{y})$. There were two bilateral and four bimodal recipients. Those who wore a hearing aid were instructed to only use their CI when carrying out music training or music listening and also when they underwent testing in the clinic. This was to control for the potential confounding effect of acoustic hearing and also to enable the recipient to focus on the sound of music through the implant, without the assistance of the HA. Participant details are provided in Table 1.

\subsection{Materials and Procedure}

2.2.1. Music Test Battery (MTB). The MTB was used to evaluate participants before and after intervention. To ensure external validity, none of the MTB stimuli was used in the MATP, and some of the MTB tasks were not specifically trained in the MATP. The MTB comprised five subtests: two pitch-ranking tests (half octave and quarter octave), three 


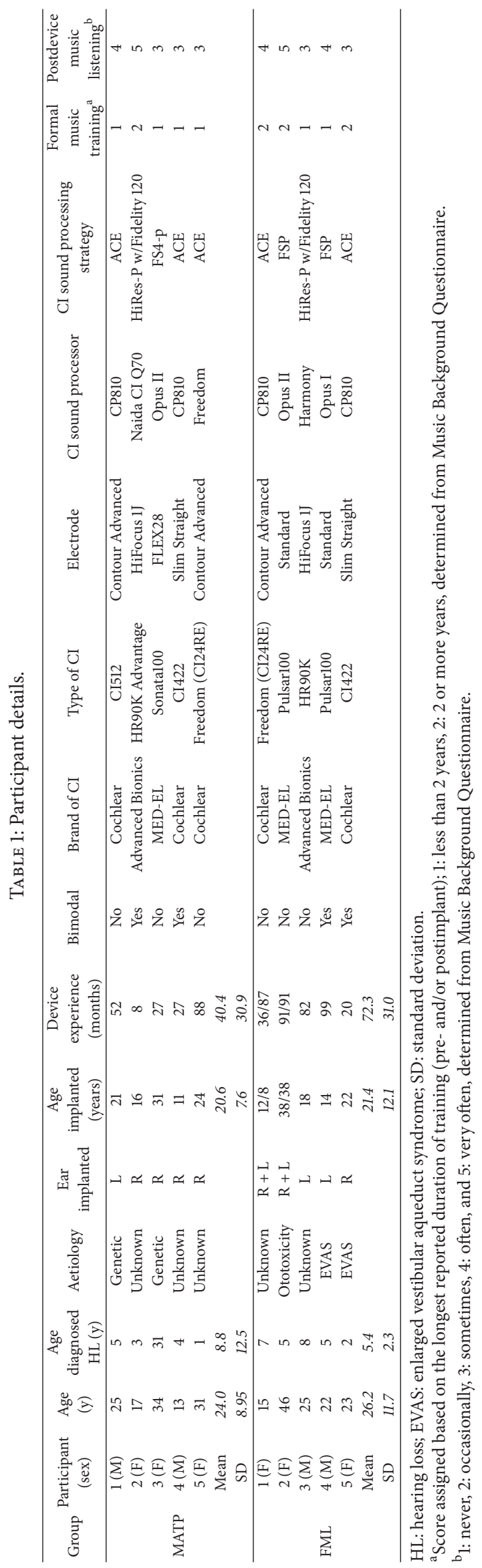


TABLE 2: Instruments and ensembles used in the identification subtests of the MTB.

\begin{tabular}{lll}
\hline Solo instruments & Music ensembles & Music styles \\
\hline Bass drum/timpani & Cello and piano duet & Classical: solo \\
Cello & Choir (four-part, a cappella) & Classical: group \\
Clarinet & Country and western band (instrumental only) & Instrumental jazz \\
Drum kit & Female singer and piano duet & Modern/pop (from 1990s onwards) \\
Female singer & Jazz band (instrumental only) & Country and Western \\
Flute & Male and female singer with piano accompaniment & Eastern \\
Guitar & Male singer and piano duet & \\
Male singer & Orchestra & \\
Piano & Percussion ensemble (varying instrumental combinations) & \\
Trumpet & Rock band (instrumental only) & \\
Violin & String quartet & \\
Xylophone & Violin and piano duet & \\
\hline
\end{tabular}

identification (ID) tests (instrument, ensemble, and style), and a music quality rating test. All tests have been validated with $\mathrm{NH}$ adults and used in prior publications with $\mathrm{CI}$ recipients and HA users [12-14, 23, 24].

The pitch-ranking test was developed by Looi et al. [12, 13 ] and requires the participant to select the higher of two notes either a half octave (six semitones) or a quarter octave (3 semitones) apart using a two-alternative forced-choice (2AFC) task response. Stimuli were sung /a/ vowels, recorded from a female singer. There were 24 items in the half-octave subtest and 32 in the quarter-octave subtest, converted to give a percent-correct score.

The instrument and ensemble ID subtests were developed and described by Looi et al. [12, 13] and examined the participants' ability to identify solo musical instruments and music ensembles, using a 12-AFC response. Prior to the first administration of the MTB, participants were given a list of instruments and ensembles which included all of the test items to check for familiarity. This was to ensure that identification abilities were being assessed rather than musical knowledge. Unfamiliar items were excluded from analyses. Stimuli comprised of 5-second extracts of 12 different instruments/ensembles, each presented four times in a randomized order (i.e., 48 items per test). Again, a percent correct score was calculated for each subtest. The list of instruments and ensembles used in the subtests is shown in Table 2. Identical versions of these two tests were used in the pre- and postintervention testing.

The style ID test was developed by Looi et al. [23], with two versions created; one version was randomly selected for the preintervention test session with the other allocated to the postintervention session for each participant. Participants were presented with 10-second musical excerpts extracted from commercially available recordings, representative of six different styles. They were asked to select the style that best matched the extract they heard (6AFC), and, with each style presented four times, a percent correct score was calculated from the 24 test items. Familiarization was again checked prior to the first administration of the test, with unfamiliar styles excluded from the analyses. The musical styles used in this test are also listed in Table 2. The test was modified from the original version to remove "pre-1960" and "1960s-80s" music as these stimuli were found to be confusing in the study by Looi et al. [14]. In order to confirm the appropriateness of the identification tests for administration with a Singaporean population, all three subtests were pilot-tested on six $\mathrm{NH}$ Singaporeans prior to the study. Their mean scores for the instrument, ensemble, and style ID subtests were 94\%, 92\%, and $90 \%$, respectively, indicating that they found the tests to be relatively easy.

The quality rating test was developed by Looi et al. [24] and subsequently adapted into a clinical software program by the Sydney Cochlear Implant Centre (SCIC). Participants were given eight pieces of music to listen to and asked to rate each of them on six different quality rating scales, according to how they sounded. The first three scales were typical visual analogue scales (VAS) with bipolar adjectives plotted equidistant from a neutral centre point with the "ideal" response being at the extreme right of the scale. These scales evaluated the qualities of "pleasantness" (unpleasant to pleasant), "naturalness" (unnatural to natural), and "richness" (tinny to rich). The other three scales are modified VAS with their midpoints labelled with the descriptor "just right." The midpoint indicated the ideal response and responses to the left or right of the midpoint indicated a deviation away from the ideal sound towards the endpoint descriptors. The three scales in this section were emptier-fuller, dullersharper, and rougher-smoother. These adjectives have been identified in previous research to best describe elements of the multidimensional concept of "timbre" [25, 26]. Four different genres were represented in the eight pieces, with each genre set containing one "common or familiar" and one "obscure or unfamiliar" piece. These are listed in Table 3. Justification for the song selection is provided in Looi et al. [24]. For the purpose of the current study, the scores from the first 3 scales were averaged for each participant (and herewith referred to as the "QR Ave1-3" score), with the second set of 3 scales averaged and referred to as the "QR Ave4-6" score.

MTB Procedure. The order of the MTB subtests was pseudorandomized, with the easier tests presented first (the halfoctave pitch-ranking test was always administered before 
TABLE 3: Pieces used for the quality rating subtest.

\begin{tabular}{lll}
\hline & Genre & Song title \\
\hline \multirow{3}{*}{ Familiar } & Modern & Raindrops Keep Falling On My Head (BJ Thomas) \\
& Classical & Serenade “Eine kleine Nachtmusik", K525, 1st movement (Mozart) \\
& Country and Western & Rhinestone Cowboy (Glenn Campbell) \\
& Common & Twinkle Twinkle Little Star \\
\hline \multirow{3}{*}{ Obscure } & Modern & Long Ago Tomorrow (BJ Thomas) \\
& Classical & Concerto in D Major K218 Allegro (Mozart) \\
& Country and Western & Do You Believe? (Dark Horizons) \\
& Common & Chu Ech On \\
\hline
\end{tabular}

the quarter-octave test and the instrument ID test before the ensemble ID test). The MACarena software [27] was used to present the MTB stimuli; MACarena automatically randomizes the presentation order of stimuli within each subtest, with responses stored in the program for later analyses. Stimuli were presented in a sound field using a single Canton Plus MX2 loudspeaker placed 0.6 metres away from the participant at 0 -degree azimuth, at individually verified comfortable presentation levels.

2.2.2. BKB-SIN Speech Perception in Noise Test. The BKBSIN is a commercially available, clinical speech-in-noise test that uses Bamford-Kowal-Bench sentences recorded in fourtalker babble. Each test items consists of two lists, each list with eight sentences with one sentence administered at each signal-to-noise ratio (SNR) of $+21,+18,+15,+12,+9,+6,+3$, and $0 \mathrm{~dB}$. Both speech and noise were presented at $60 \mathrm{dBHL}$ in the free-field via the FBT clinical audiometry loudspeaker placed one metre away from the listener, at 0-degree azimuth.

Specific scoring instructions are provided in the software, but an SNR-50 score is obtained which represents the signalto-noise ratio for a $50 \%$ correct score whereby a lower score indicates a better result.

2.2.3. Questionnaires. Two questionnaires were administered in this study. The Music Background Questionnaire was adapted from Looi et al. [13] and used to gather information on participants' formal music training, self-rated music ability, and music listening habits. In addition, an evaluation questionnaire was given to participants at the end of the study to obtain feedback and ratings of self-perceived benefits and changes to their music listening as a result of undertaking the FML or the MATP.

2.2.4. Music Appreciation Training Program (MATP). The MATP is a take-home, computer-based auditory training program, developed by Looi et al. [14] and subsequently modified for this study based on the feedback reported in the former study [14]. Unlike existing training programs published in the literature, this training program focused on music appreciation and enjoyment, rather than just perceptual accuracy. Therefore, as opposed to having analytic, psychoacoustic tasks such as pitch-ranking or melodic contour identification, this program started with instrument identification before progressing to music ensemble identification and then music style/genre identification. Perceiving musical styles requires a listener to integrate several musical elements together (e.g., rhythm, pitch, timbre, and even dynamics) as opposed to focusing on only one of these elements in isolation. All of the stimuli were excerpts of "real-world" music either played by live instruments or extracted from commercially available compact disc recordings.

A detailed description of the original program and the training tasks is provided in Looi et al. [14]; however, a short overview is provided here. The original program comprised five modules, with modules 1 to 4 being the true training modules and module 5 containing extra optional tasks, tips, and resources. Each module (except for 5) comprised three phases - a teaching phase, a training phase, and a self-testing phase. In the teaching phase, 10 samples of each instrument, ensemble, or music style were provided for the trainee to listen to, accompanied by information on the specific extract and the sounds that were being played. Once the trainee felt that they were sufficiently familiar with the different stimuli, they then progressed to the training phase where they were then required to differentiate between the different stimuli using a closed-set format. This phase incorporated an adaptive algorithm whereby the number of options presented on screen changed according to their response accuracy (i.e., increased number of options if the trainee correctly identified 3 consecutive stimuli, or 3 out of 4 consecutive trials, or the reverse situation for incorrect responses). In this phase, on-screen feedback was provided as to whether responses were correct or incorrect, along with the opportunity to rehear stimuli. The self-testing phase enabled the trainee to monitor their progress with 20 randomly selected stimuli being presented in a closed-set identification test, where all of the possible options were displayed on screen. After completing the test, both the total score as well as the results for each individual item were provided to encourage trainees to return to the teaching phase and practise with those stimuli they had difficulty with. The program automatically recorded scores in both the training and self-testing phase and datalogged information such as the time spent training, dates of training, modules used, and time spent with each module.

The modified version preserved most of the functionality of the original program, including data logging, but made improvements to the speed and user interface. The advanced module and the "additional information/resources" module from the original program were also removed as the duration 
TABLE 4: Stimuli included in each module of the MATP.

\begin{tabular}{|c|c|c|}
\hline $\begin{array}{l}\text { Module } 1 \\
\text { Solo } \\
\text { instruments }\end{array}$ & $\begin{array}{l}\text { Module } 2 \\
\text { Ensembles }\end{array}$ & $\begin{array}{l}\text { Module } 3 \\
\text { Musical styles }\end{array}$ \\
\hline Piano & Brass band & Classical: solo \\
\hline Violin & Choir & Classical: small group \\
\hline Cello & Duets: instrumental & Classical: large group \\
\hline Flute & Duets: voice + piano & Country and western \\
\hline Clarinet & Instrument + orchestra & Eastern \\
\hline Trumpet & Jazz band & Jazz \\
\hline Trombone & Orchestra & $\begin{array}{l}\text { Modern/pop (from } \\
\text { 1990s onwards) }\end{array}$ \\
\hline Xylophone & Rock band & \\
\hline Drum kit & String quartet & \\
\hline Guitar & Voice + orchestra & \\
\hline \multicolumn{3}{|l|}{ Male singer } \\
\hline Female singer & & \\
\hline
\end{tabular}

for training was shorter in this study. The three remaining modules were (1) solo instruments; (2) musical ensembles, and (3) musical styles. Another modification from the original program was that two music styles, "1960s-1980s" and "old-time music (1950s and earlier)," were removed, as Looi et al. [14] reported that these stimuli were more likely to have been confused due to music knowledge (i.e., time/decade) rather than actual perceptual accuracy. Table 4 provides a list of the instruments, ensembles, and styles incorporated into the respective modules. It should also be noted that the stimuli in the MATP was different to that used in the $\mathrm{MTB}$, in order to ensure that any training benefits were not attributable to task-specific learning effects. Different artists, groups, recordings, and excerpts were used. The MTB was developed separately and largely prior to the MATP used in this study.

Training Procedure. Participants were asked to train for a total of eight weeks, four times a week for 30 minutes per session. They were instructed to spend two weeks on each of modules one, two, and three (in that order), followed by two weeks of "own choice" revision. The reason for standardising this module order was that the modules were inherently progressive; that is, single instrument stimuli should be easier than music ensembles, with music ensembles naturally leading to musical styles. Participants in the MATP group were given a set of Logitech LS21 computer speakers and instructed to plug these speakers into their computer and use them for training. A user manual containing instructions on how to install, run, and use the MATP was also provided, with a training diary at the back for participants to record their progress.

2.2.5. Focused Music Listening (FML) Task. Unlike existing music training studies where the control group did not have anything to do, in this study a music listening task was developed to account for the "music exposure" variable. This task involved listening to music for the same amount of time that the MATP group spent training (i.e., 30 minutes, four times a week). However, to avoid passive music listening where the participant is engaged in other activities with music playing in the background, participants were given a series of questions to think about for each piece. These questions covered the features of the music (e.g., "what was the mood of the piece?"), the instrumentation used (e.g., "how many instruments or voices did you hear?"), and their subjective appraisal of the music (e.g., "what did you like or not like about the piece?").

FML Procedure. The quantity of FML was the same as the training time for the MATP group, that is, four 30-minute sessions per week, for eight weeks. However, unlike the MATP group, no restrictions or guidelines were set on the type of music they could listen to; the only requirement was that they were to listen to each piece at least two times. Further, no stipulation was made as to the listening mode to use or music genre(s) to try to enable participants to freely explore and develop their personal music preferences and needs. A guide containing the task instructions, questions, and a music listening diary to record their sessions was provided.

2.3. Overall Procedure. The study was divided into three periods-a 2 -week to 4 -week control period, 8 weeks of training (or music listening), and a follow-up test session between 4 to 8 weeks $(M=5.6$ weeks; SD $=1.65)$ after the cessation of training. The MTB and BKB-SIN were administered four times per participant-before and after the control period, immediately after the intervention period, and finally between 4 to 8 weeks after intervention. The Music Background Questionnaire was completed at the first session and the evaluation questionnaire in the third session. For all of the testing, participants were asked to set their devices to their everyday program and settings and not to change this during the course of testing. They were also asked to utilize these same settings at the subsequent test sessions.

\section{Results}

All 10 participants completed the study; however, one MATP participant trained for seven weeks due to scheduling issues. Mann-Whitney $U$ tests showed no significant difference between the MATP and FML groups for the participant factors of age, age at implantation, device experience, formal music training, or postdevice music listening.

3.1. Training Details. The MATP data logs showed that on average the MATP participants completed 618 of the 960 minutes requested. It should be noted, though, that two of the five participants in this group reported forgetting to save some of the training sessions to the data log. For the FML group, an average of 925 minutes of FML was recorded in their diaries. In this group, FML5 did not submit their listening diary and was not included in this analysis. These diaries were also analysed to determine the musical preferences of 
TABLE 5: MTB and BKB-SIN scores of the two groups over all three visits.

\begin{tabular}{|c|c|c|c|c|c|c|c|c|}
\hline & \multicolumn{4}{|c|}{ MATP group } & \multicolumn{4}{|c|}{ FML group } \\
\hline & 1st visit & 2nd visit & 3rd visit & 4th visit & 1st visit & 2nd visit & 3rd visit & 4th visit \\
\hline \multicolumn{9}{|l|}{ MTB (\% correct) } \\
\hline Pitch-ranking $1 / 2$ octave & $71.7(15.4)$ & $71.7(19.0)$ & $68.3(19.7)$ & $71.7(22.1)$ & $71.7(7.45)$ & $70.0(18.7)$ & $71.7(12.3)$ & $70.8(15.9)$ \\
\hline Pitch-ranking $1 / 4$ octave & $67.5(18.6)$ & $61.3(27.0)$ & $60.6(23.5)$ & $60.6(28.0)$ & $54.4(15.7)$ & $60.6(17.9)$ & $54.4(21.9)$ & $57.5(14.1)$ \\
\hline Instrument ID & $60.6(15.4)$ & $64.7(14.6)$ & $70.8(16.0)$ & $72.4(17.7)$ & $60.8(10.6)$ & $68.8(12.6)$ & $72.1(8.79)$ & $69.2(13.1)$ \\
\hline Ensemble ID & $39.3(15.8)$ & $48.1(19.3)$ & $47.9(19.0)$ & $40.8(20.7)$ & $41.3(2.62)$ & $41.5(10.6)$ & $43.8(7.24)$ & $41.4(6.3)$ \\
\hline Style ID & $39.8(6.38)$ & $56.5(22.0)$ & $46.7(24.5)$ & $49.2(28.6)$ & $53.7(17.5)$ & $45.5(22.1)$ & $40.0(23.5)$ & $52.2(20.4)$ \\
\hline QR Ave1-3 ${ }^{\mathrm{a}}$ & $54.5(7.79)$ & $63.5(9.89)$ & $68.8(9.88)$ & $63.6(15.7)$ & $63.1(12.3)$ & $60.6(16.7)$ & $59.7(17.9)$ & $52.2(27.7)$ \\
\hline QR Ave4-6 ${ }^{\mathrm{b}}$ & $5.0(5.26)$ & $5.5(5.12)$ & $14.6(18.0)$ & $9.2(18.8)$ & $5.6(7.16)$ & $7.1(10.9)$ & $7.5(5.29)$ & $2.0(13.4)$ \\
\hline \multicolumn{9}{|l|}{ BKB-SIN (dB) } \\
\hline SNR- $50^{c}$ & $16.20(5.05)$ & $15.15(5.02)$ & $15.30(4.85)$ & $15.0(4.7)$ & $16.60(3.54)$ & 15.15 (1.86) & 15.85 (2.97) & $16.0(3.8)$ \\
\hline
\end{tabular}

Values shown are the mean (with standard deviation in parentheses).

${ }^{a}$ Avel-3 scores range from 0 to 100 , with 100 being the best response.

${ }^{\mathrm{b}}$ Ave4-6 scores indicate deviation from 0 , which is the best response (the scale ranged from -50 to +50 , but absolute values were used for this analysis).

${ }^{\mathrm{c}}$ Scores are SNR-50, that is, the signal-to-noise ratio, where a $50 \%$ correct score would be obtained for the sentence test material. A lower value indicates better speech perception in noise.

the participants. The pieces of music listed in the diaries were categorized into the following genres: classical, English pop, Chinese pop, miscellaneous instrumental, and others. The most popular genre was English pop, selected 37\% of the time across all FML participants. This was followed by classical (27\%), Chinese pop (20\%), and miscellaneous instrumental $(13 \%)$.

3.2. Control Period (Sessions 1 and 2). Mean scores for each of the tests for each visit are summarized in Table 5.

There were no significant differences between the baseline (Session 1) scores for the MATP and FML group for any of the MTB subtests (Mann-Whitney $U$ tests; $p>0.05$ ). Onesample $t$-tests were carried out to see if the pitch-ranking scores (both groups combined) differed significantly from the chance score of $50 \%$. For the quarter-octave interval size, the combined group's mean score of $61 \%$ did not differ significantly from the chance of $50 \%(t(9)=1.96, p=0.081)$. Scores for the half-octave interval were significantly better than chance. For the ID tests, the combined group scored the highest for instrument ID $(M=60.7 \%)$, followed by style ID $(M=46.7 \%)$, and scored the lowest on ensemble ID ( $M=$ $40.3 \%)$. Pairwise comparisons with a Bonferroni correction showed these differences to be statistically significant $\left(\chi^{2}(2)\right.$ $=9.80, p=0.007)$. There was also no significant difference between the baseline BKB-SIN scores of the two groups (Mann-Whitney $U$ tests).

Spearman's rho calculations showed no significant correlations between the participant factors of age, device experience, music training and music listening levels (as determined from the music background questionnaire and reported in Table 1), and MTB scores. For these analyses, the MTB subtests were grouped as follows: pitch-ranking tasks (the mean of each participant's quarter- and half-octave pitch-ranking scores); ID tasks (mean of their instrument, ensemble and style ID scores); QR Avel-3 (mean of the quality rating scores for scales 1-3); QR Ave4-6 (mean of the quality rating scores for scales 4-6).
When the scores of the first and second visits were compared to determine if there was a significant task-related learning effect, no significant difference was found for any of the tests (the Wilcoxon sign-rank tests). Accordingly, these two scores were averaged and combined into a "pretraining" score for each participant. In the subsequent sections, the effect of the intervention (MATP or FML) will be assessed by comparing this pretraining score to the score on the third ("posttraining") visit.

3.3. Posttraining Results (Session 3). The Wilcoxon sign-rank tests between the pretraining score and the session 3 score showed that, for the MATP group, there was a significant improvement in the instrument ID scores $(z=-2.02$, $p=0.043)$. In the FML group, no significant increases were observed, with the improvement in ensemble ID scores approaching significance $(z=-1.75, p=0.080)$. No significant changes in quality ratings were observed in either group for either the QR Avel-3 or QR Ave4-6 scores, although the increase in the QR Avel-3 ratings after training was approaching significance $(z=-1.75, p=0.080)$ for the MATP group. BKB-SIN scores did not change significantly for either group from before to after intervention.

To compare the effects of computer-based training to focused music listening, "difference scores" (i.e., posttraining score minus pretraining score) were calculated for each test. These are shown in Table 6. Mann-Whitney $U$ tests were conducted to compare these "difference scores" between the MATP and FML groups to determine if one intervention led to significantly greater levels of improvement than the other. The analysis showed that the change in QR Avel-3 ratings for the MATP group was nearly significantly greater than that of the FML group $(U=4.00, p=0.07)$.

3.4. Follow-Up Session Scores (Session 4). Wilcoxon signranks tests were conducted to compare the Session 3 to Session 4 scores to determine whether any changes as a result of the MATP or FML were sustained after the intervention 
TABLE 6: Difference scores of each test for both groups.

\begin{tabular}{lcc}
\hline & MATP group & FML group \\
\hline MTB & & \\
Pitch-ranking 1/2 octave & -3.327 & 0.834 \\
Pitch-ranking 1/4 octave & -3.742 & -3.124 \\
Instrument ID & 8.208 & 7.293 \\
Ensemble ID & 4.229 & 2.447 \\
Style ID & -1.461 & -9.584 \\
QR Avel-3 & 9.76 & -2.135 \\
QR Ave4-6 & 9.287 & 1.201 \\
BKB-SIN (dB) & & \\
SNR-50 & -0.375 & 0.025 \\
\hline
\end{tabular}

"Positive values for the "QR Ave4-6" and "BKB-SIN SNR-50" indicate that the scores worsened after the training period.

was ceased. Results showed that the ensemble ID and QR Ave4-6 scores were significantly lower in session 4 than session 3. No other significant changes were observed.

A graphical representation of each participant's mean score for each test at the three time points (pretraining, session 3 , and session 4), along with the two group's means for each test and time point, is provided in Figure 1. As is evident in the figure, there was a lot of individual variability in the data.

3.5. Perceived Benefits of Training Period. A summary of the perceived benefits, as reported in the evaluation questionnaire is shown in Figure 2. All of the participants in this study found their respective intervention useful, with the average benefit reported being 3.3 for both groups (on a scale from 1 to 5). For the MATP group, the greatest perceived benefit was improved ability to recognize instruments or ensembles, as well as music sounding more pleasant. For the FML group, improved ability to recognize instruments or ensembles and music sounding more natural were the highest rated benefits. Eight of the ten participants (four in each group) felt that they could continue to improve their music perception skills as a result of participating in this study.

\section{Discussion}

A significant change was observed between pre- and posttraining scores for the instrumental ID subtest in the MATP group, suggesting that the music training program helped this group of recipients improve their timbre perception. This improvement was sustained at the follow-up appointment, one to two months after training has ceased. This was in contrast to the findings by Looi et al. [14] where the MATP led to improved subjective quality ratings, but not identification scores. One of the reasons for this difference could be that the participants in the earlier [14] study were a lot older than the participants in the current study (60.2 years in [14] compared to 25.1 years in the current study). Looi et al. [14] found a strong negative correlation between the age of participants who trained with the MATP and the overall perceived benefit. It is possible that age might have an effect on actual benefit, with younger participants being more inclined to show improvements in posttraining scores due to cognitive and/or attention factors, and neurological changes related to aging. Another difference of note is the baseline performance of the participants in the two studies. In the initial study [14], the pretraining scores for all three of the MTB ID subtests were significantly higher than the first-visit scores recorded in this study. Hence, it may be that some of the participants in the earlier study already had reasonable timbre perception skills and therefore less room for improvement for that cohort. Further, the current cohort's music experience levels were lower than those of [14], giving them more potential to improve on the ID tests after training with the MATP. It is worthwhile restating that the stimuli in the MATP were completely different from the stimuli in the MTB, demonstrating that music training can be generalized to different stimuli and musical instruments.

While there was significant improvement found for the instrument ID subtest, there was no significant changes for the ensemble or style ID tests. The lack of improvement on the ensemble and style ID tests for this group could be due to a few reasons. Firstly, discriminating between solo instruments is an easier task than discriminating between multiple instruments playing simultaneously and/or different styles of music. To perform well in the ensemble ID test, one has not only to be able to identify single instruments, but also be able to recognize them when they are being played with other instruments simultaneously. The discrimination between music styles is challenging in that not only does it requires the listener to identify various instruments playing together, but also it requires some musical knowledge in that the listener would need to have an awareness of what instrumentation, rhythmic and/or harmonic features are characteristic to a style. For example, modern pop music often has guitars and drums, and a pervasive consistent beat; jazz music often uses saxophones, clarinets, or trumpets and is associated with swing or blues rhythms; and eastern music is based largely on pentatonic scales. Being able to identify different styles of music requires both perceptual accuracy for the key elements of the musical stimuli and knowledge of the features of the styles. The higher difficulty levels of the ensemble and style ID tests are also reflected in the first-visit scores of the cohort; scores were higher for the instrument ID subtest than for the other two subtests.

Time spent on training was another factor which could have contributed to the improvement in instrument ID scores but not in the other two ID subtests. Data logs from the MATP group showed that collectively, participants spent the most time training on the solo instruments module and the least time training on the musical styles module. It is interesting to note that the two subjects who spent the most time in the musical ensembles module, MATP2 and MATP5, both showed improvements on the posttraining scores for the ensemble ID subtest. For example, MATP2 scored $68.8 \%$ after training, an improvement from her score of $52.1 \%$ before training. She also reported that she spent more sessions training in that module as she found it more challenging to differentiate between the ensembles than between single instruments. 


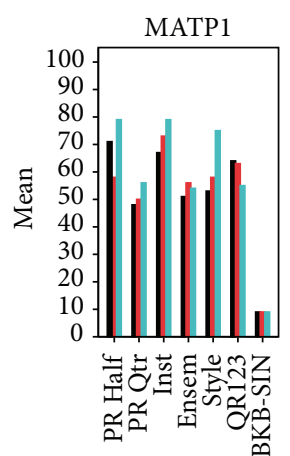

Test
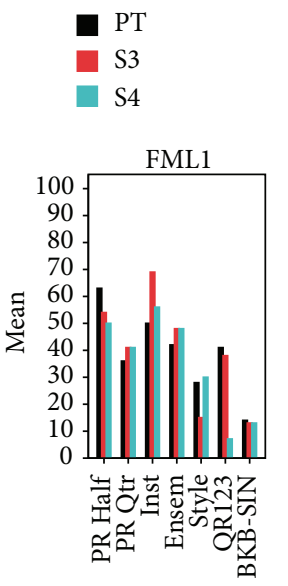

Test

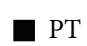

S3

S4

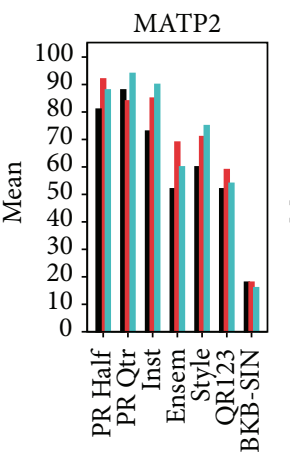

Test

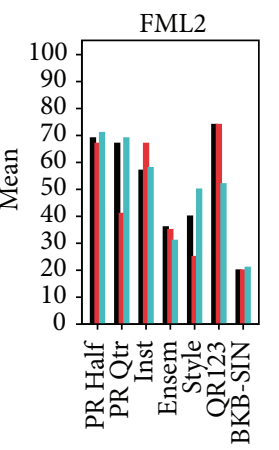

Test

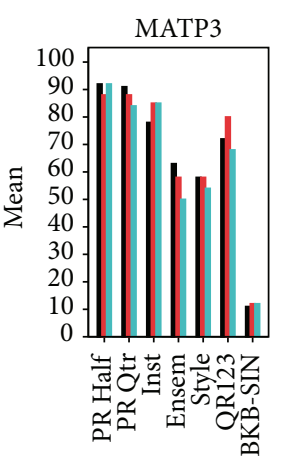

Test

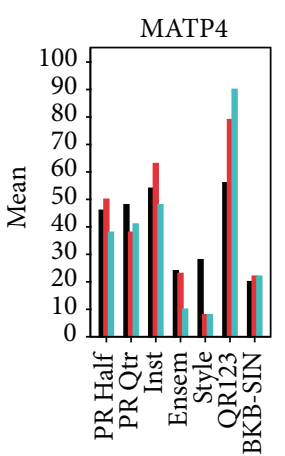

Test

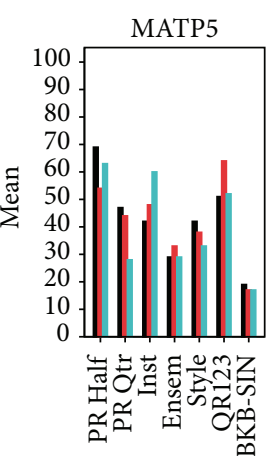

Test

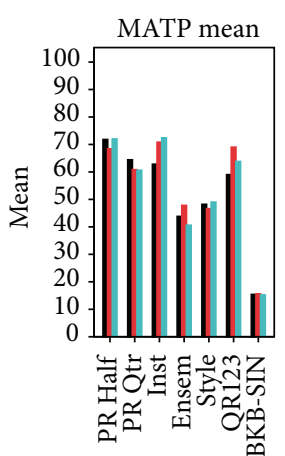

Test

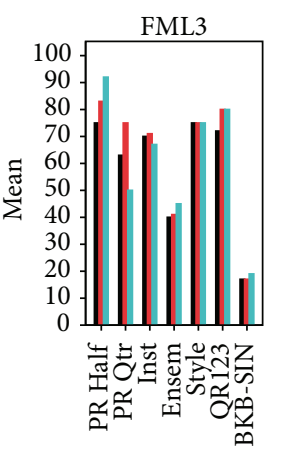

Test

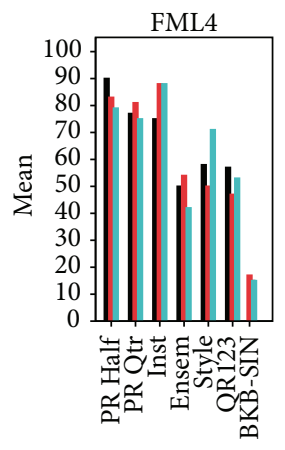

Test

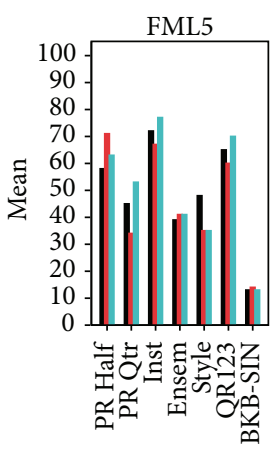

Test

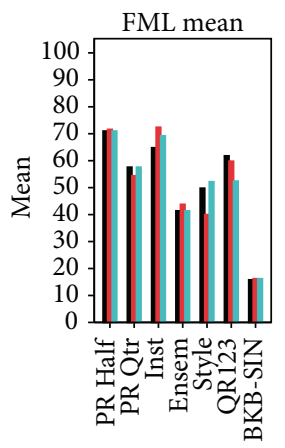

Test

FIGURE 1: Each participant's mean score for each of the MTB subtests at the three time points (pretraining, session 3, and session 4), along with the two group's means for each subtest and time point. The top panel is the MATP group, with the bottom panel being the FML group. The last graph in each panel is the mean scores for that group. PT: pretraining mean scores (i.e., average of sessions 1 and 2 scores). S3: session 3 mean scores. S4: session 4 mean scores. The $y$-axis is numbered from 0 to 100 . This represents either a \% correct score (for the pitch-ranking and 3 identification tests), a score/100 for the QR Avel-3 subtest, and the SNR-50 score for the BKB-SIN test (the signal-to-noise ratio where the recipient would have obtained $50 \%$ correct score for the sentence test material). Note for the BKB-SIN test, a lower score indicates a better result. The QR Ave4-6 score is not presented in this figure, as the scoring is neither linear or on a scale from 0 to 100 . The $x$-axis are the different MTB subtests. PR Half: pitch-ranking half octave; PR Qtr: pitch-ranking quarter-octave; Inst: instrument identification; Ensem: ensemble identification; Style: style identification; QR123: quality rating test, average of scales 1-3; BKB-SIN: BKB sentence in noise test (SNR-50 score).

Improvements in music quality ratings were also seen for the MATP group where ratings for the first three scales, as represented by the "QR Avel-3" score, increased over the visits, with the difference between the pre- and posttraining scores approaching significance $(p=0.08)$. This is in keeping with the findings of Looi et al. [14] where a significant difference in music quality ratings was seen in the group that utilized the MATP. This improvement was also sustained at the follow-up visit, in the current study.

For the FML group, no statistically significant pre- to postimprovements in scores were observed. However, it is worth noting that substantial improvements in the instrument and ensemble ID scores were observed for some participants (e.g., FML1 and FML4 (see Figure 1)), which may indicate that FML could help with music perception to some extent or at least for some individuals. A larger sample size is necessary to investigate this further and to ascertain whether there may be any particular predictive factors that could help determine which recipients may obtain benefit. Additionally, it may be that providing more structure and guidance to the FML program may also improve outcomes (e.g., by having session plans accompanied by written notes and/or visual resources such as pictures or a DVD).

Overall, the data from this group of recipients suggests that structured music training significantly improves music perception; however, FML may provide benefit for some individuals. One clinical application of this is that although a structured music training program seemingly offers the most benefit to CI recipients, FML could be offered as an alternative given there are currently no commercially available music training programs for adult recipients. The clinician and recipient could work together to structure a music listening 


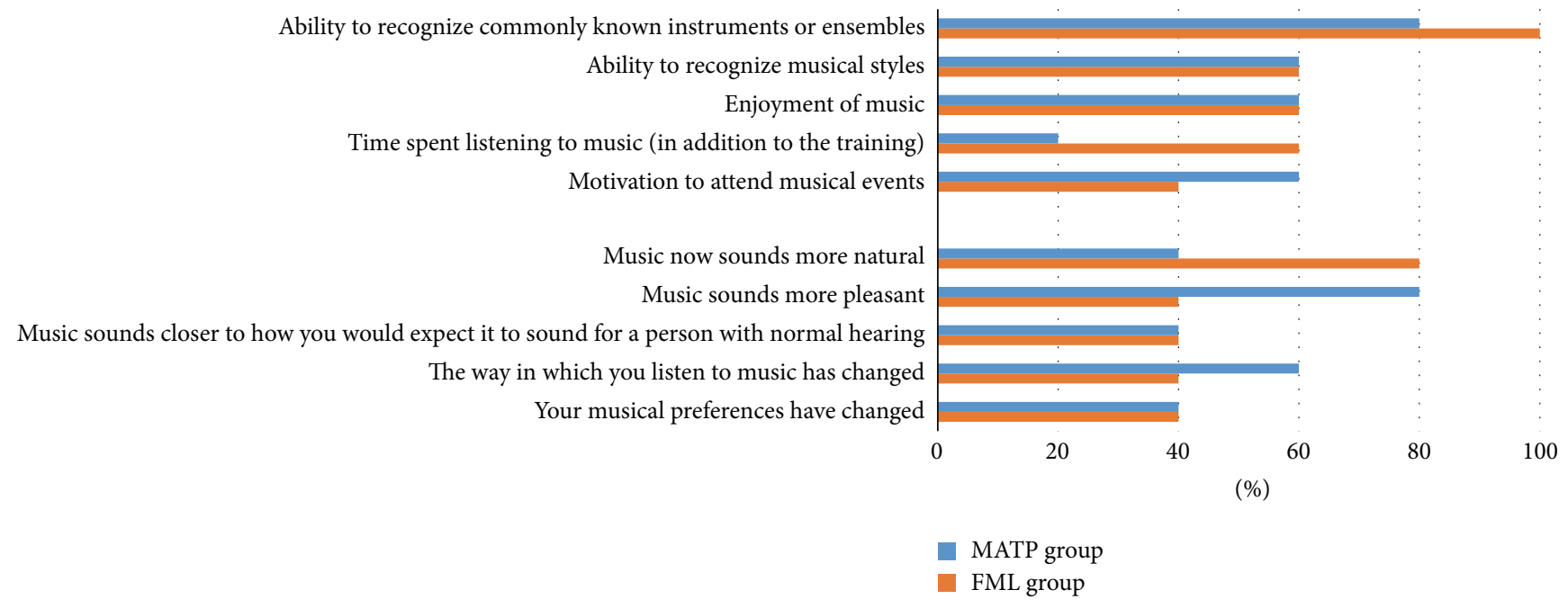

FIGURE 2: Subjective benefits, as reported in the evaluation questionnaire. The first set of questions (in the top section) started with "Do you feel that the MATP/FML task has helped to increase your..." The second set of questions (in the lower section) started with "Since carrying out the MATP/FML task, do you feel that...." The bars correspond to the \% of participants who responded "noticeably" or "very much so" (on a 5-point Likert Scale).

program that involves both analytic and synthetic musicbased listening tasks, whilst accounting for the patient's musical preferences, interests, motivation, and available time. Written notes and/or visual material could supplement the program, and guidance provided around what to listen to, and what to listen for, may be of further help. What is most important in a FML task is that the recipient is engaged in the task, attentive to the music they are listening to, and analytic in their reflections of what they are hearing. Providing structured guidelines and specific questions related to the music and getting the recipient to make comparisons between the auditory information heard are critical to the process, rather than just listening to the music passively or making simplistic (e.g., like or dislike) judgments about the music.

Although research has indicated the presence of crossdomain plasticity and the mechanisms and information that contribute to pitch perception (e.g., temporal fine structure) are also applicable to auditory segregation which is critical for speech-in-noise perception, neither group's speech-in-noise perception improved after intervention. It may be that crossdomain plasticity requires a longer period of training and/or that both the MATP and FML tasks in this study were more synthetic and timbre-based, rather than analytic pitch-based training tasks (e.g., pitch-ranking). That is, synthetic timbrebased tasks may not generalize to a highly analytic pitchspecific perceptual task, or at least not with the quantity of training used in this study. These findings were also found by Looi et al. [14] where pitch-ranking scores did not improve after 10 weeks of using the MATP program.

Interestingly, both groups subjectively felt that they benefitted from their respective interventions, irrespective of whether or not this benefit translated to improved scores on the objective tests. All ten participants reported that their ability to recognize commonly known instruments or ensembles had changed either "noticeably" or "very much."
Seven (three from the MATP group and four from the FML group) also reported similar improvements for music style recognition. Four of the five participants in the MATP group felt that music sounded more pleasant and that they enjoyed music more, and four of five of the FML group felt that music sounded more natural. Although these perceived benefits were not entirely reflected in the MTB scores, these subjective benefits are still important as they may be the "real-world" changes that a recipient may notice or could even just provide a recipient with more confidence to listen to music or be more actively engaged in musical activities. Obviously, it must also be considered that participants provided positive feedback to "please" the researcher.

As mentioned earlier, FML could be offered as an option for recipients wanting to improve their music listening, particularly given that a music training program is not commercially available to all CI recipients at present. Another approach would be to have a combination of computer-based training and FML. Compliance in the MATP group was lower than in the FML group, which could be reflective of higher motivation for the less structured, more individualized FML task. Having a combination of both approaches may help to increase motivation and compliance, for example, using a training program at the start to provide immediate feedback on the stimuli being played, followed by FML to enable the recipient to build on this and/or tailor their rehabilitation to better meet their preferences. It should be kept in mind that existing research has shown that incidental exposure to music and time with CI do not result in improved music perception $[9,21]$. Long term, sustained improvement in music listening for $\mathrm{CI}$ recipients requires ongoing work and persistence with music-related tasks. A short-term, one-off training program undertaken for a few weeks may not lead to long-term, ongoing improvements in music perception for CI recipients. Therefore, working with a recipient to determine an approach which they would be more likely to persevere 
with and adopt for a longer period of time would have more potential of success and benefit than a short, intense burst with no follow-up work.

\section{Conclusions}

Overall, the structured music training program provided better outcomes than FML, with a significant difference observed for the MATP group on the instrument ID tasks, as well as the first three quality rating scales. However, although statistically significant improvements were not obtained for the FML group, higher scores were observed for some participants after FML for the instrument and ensemble ID tasks, and compliance with the task was substantially better than for the MATP group. These results suggest that although a music training program may help adult recipients to improve their music perception, in lieu of a training program, FML could be offered as an alternative, particularly if the FML was structured to ensure active, focused, analytic listening with appropriate questions and guidance from a clinician. Alternatively, a combination of the two approaches could be adopted, with a training program used initially to provide feedback and structure in the initial "learning" or "reintroduction" phase, followed by FML over a longer term to build on gains obtained from the training program, and allow greater individualization and relevance for the recipient.

\section{Conflict of Interests}

The authors declare that there is no conflict of interests regarding the publication of this paper.

\section{Acknowledgments}

The authors would like to thank Mr. Joe Chee for all of the modifications made to the MATP software. In addition, they thank staff at the National University Hospital Audiology Department for help with recruitment, Dr. Wai Kong Lai for the MACarena software used for testing, and the participants who devoted a lot of time and effort to this study. This study was funded equally by Cochlear Ltd., Advanced Bionics, and MED-EL, although the manufacturers had no input into the study design and were not provided with copies of the results.

\section{References}

[1] Y. Y. Lee, M. F. Chan, and E. Mok, "Effectiveness of music intervention on the quality of life of older people," Journal of Advanced Nursing, vol. 66, no. 12, pp. 2677-2687, 2010.

[2] A. J. Lonsdale and A. C. North, "Why do we listen to music? A uses and gratifications analysis," British Journal of Psychology, vol. 102, no. 1, pp. 108-134, 2011.

[3] A. C. North, D. J. Hargreaves, and S. A. O’Neill, “The importance of music to adolescents," British Journal of Educational Psychology, vol. 70, no. 2, pp. 255-272, 2000.

[4] T. Schäfer, P. Sedlmeier, C. Städtler, and D. Huron, "The psychological functions of music listening," Frontiers in Psychology, vol. 4, article 511, Article ID Article 511, 2013.
[5] T. Hays and V. Minichiello, "The contribution of music to quality of life in older people: an Australian qualitative study," Ageing and Society, vol. 25, no. 2, pp. 261-278, 2005.

[6] R. A. R. MacDonald, "Music, health, and well-being: a review," International Journal of Qualitative Studies on Health and Wellbeing, vol. 8, no. 1, Article ID 20635, 2013.

[7] C. J. Limb and A. T. Roy, "Technological, biological, and acoustical constraints to music perception in cochlear implant users," Hearing Research, vol. 308, pp. 13-26, 2014.

[8] V. Looi, "The effect of cochlear implantation on music perception a review," Otorinolaryngol, vol. 58, no. 4, pp. 169-190, 2008.

[9] V. Looi, K. Gfeller, and V. Driscoll, "Music appreciation and training for cochlear implant recipients: a review," Seminars in Hearing, vol. 33, no. 4, pp. 307-334, 2012.

[10] H. McDermott, "Music perception," in Auditory Prostheses: New Horizons, F. G. Zeng, A. N. Popper, and R. R. Fay, Eds., pp. 305339, Springer Science \& Business Media, 2012.

[11] K. Gfeller, J. Oleson, J. F. Knutson, P. Breheny, V. Driscoll, and C. Olszewski, "Multivariate predictors of music perception and appraisal by adult cochlear implant users," Journal of the American Academy of Audiology, vol. 19, no. 2, pp. 120-134, 2008.

[12] V. Looi, H. McDermott, C. McKay, and L. Hickson, “The effect of cochlear implantation on music perception by adults with usable pre-operative acoustic hearing," International Journal of Audiology, vol. 47, no. 5, pp. 257-268, 2008.

[13] V. Looi, H. McDermott, C. McKay, and L. Hickson, "Music perception of cochlear implant users compared with that of hearing aid users," Ear and Hearing, vol. 29, no. 3, pp. 421-434, 2008.

[14] V. Looi, J. King, and R. Kelly-Campbell, "A music appreciation training program developed for clinical application with cochlear implant recipients and hearing aid users," Seminars in Hearing, vol. 33, no. 4, pp. 361-380, 2012.

[15] B. A. Wright and Y. Zhang, "A review of the generalization of auditory learning," Philosophical Transactions of the Royal Society of London, Series B: Biological Sciences, vol. 364, no. 1515, pp. 301-311, 2009.

[16] N. Kraus and B. Chandrasekaran, "Music training for the development of auditory skills," Nature Reviews Neuroscience, vol. 11, no. 8, pp. 599-605, 2010.

[17] A. Parbery-Clark, D. L. Strait, and N. Kraus, "Contextdependent encoding in the auditory brainstem subserves enhanced speech-in-noise perception in musicians," Neuropsychologia, vol. 49, no. 12, pp. 3338-3345, 2011.

[18] D. L. Strait, A. Parbery-Clark, E. Hittner, and N. Kraus, "Musical training during early childhood enhances the neural encoding of speech in noise," Brain and Language, vol. 123, no. 3, pp. 191201, 2012.

[19] A. D. Patel, "Can nonlinguistic musical training change the way the brain processes speech? The expanded OPERA hypothesis," Hearing Research, vol. 308, pp. 98-108, 2014.

[20] W. R. Drennan, J. J. Oleson, K. Gfeller et al., "Clinical evaluation of music perception, appraisal and experience in cochlear implant users," International Journal of Audiology, vol. 54, no. 2, pp. 114-123, 2015.

[21] K. Gfeller, D. Jiang, J. J. Oleson, V. Driscoll, and J. F. Knutson, "Temporal stability of music perception and appraisal scores of adult cochlear implant recipients," Journal of the American Academy of Audiology, vol. 21, no. 1, pp. 28-34, 2010. 
[22] B. Philips, B. Vinck, E. De Vel et al., "Characteristics and determinants of music appreciation in adult CI users," European Archives of Oto-Rhino-Laryngology, vol. 269, no. 3, pp. 813-821, 2012.

[23] V. Looi, J. King, and R. Kelly, "Validation of a music style identification test for cochlear implant and hearing aid users," in Presentation at the 8th Asia Pacific Symposium on Cochlear Implants and Related Sciences, pp. 25-28, Daegu, South Korea, October 2011.

[24] V. Looi, P. Winter, I. Anderson, and C. Sucher, "A music quality rating test battery for cochlear implant users to compare the FSP and HDCIS strategies for music appreciation," International Journal of Audiology, vol. 50, no. 8, pp. 503-518, 2011.

[25] G. von Bismarck, "Sharpness as an attribute of the timbre of steady sounds," Acustica, vol. 30, no. 3, pp. 159-172, 1974.

[26] G. von Bismarck, "Timbre of steady sounds: a factorial investigation of its verbal attributes," Acustica, vol. 30, no. 3, pp. 146159, 1974.

[27] W. K. Lai and N. Dillier, "Macarena: a flexible computerbased speech testing environment," in Proceedings of the 7th International Cochlear Implant Conference, Manchester, UK, September 2002. 


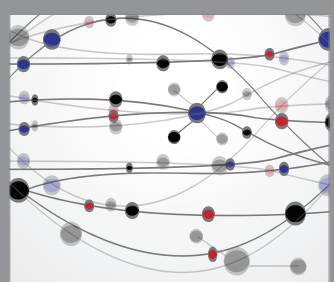

The Scientific World Journal
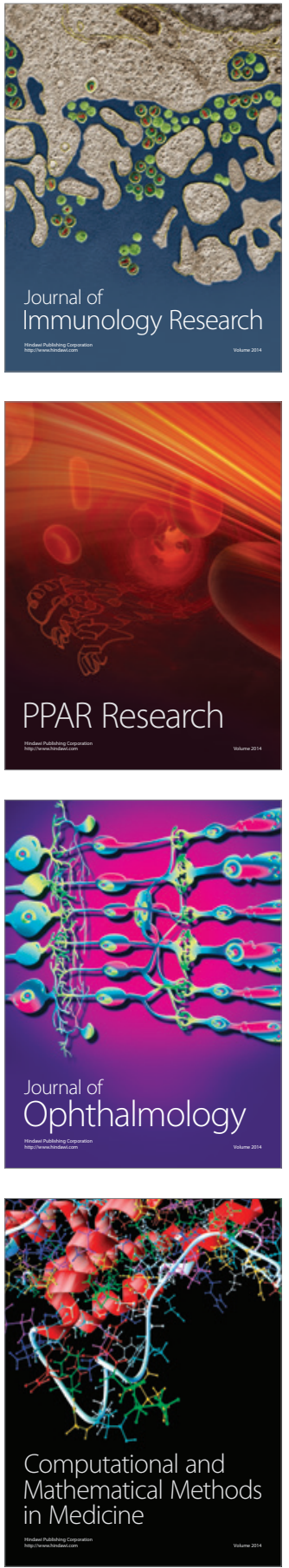

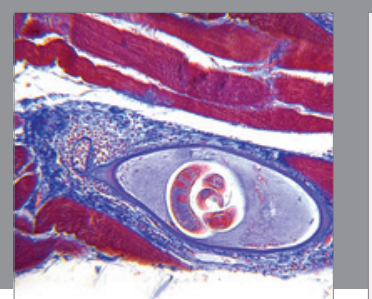

Gastroenterology Research and Practice

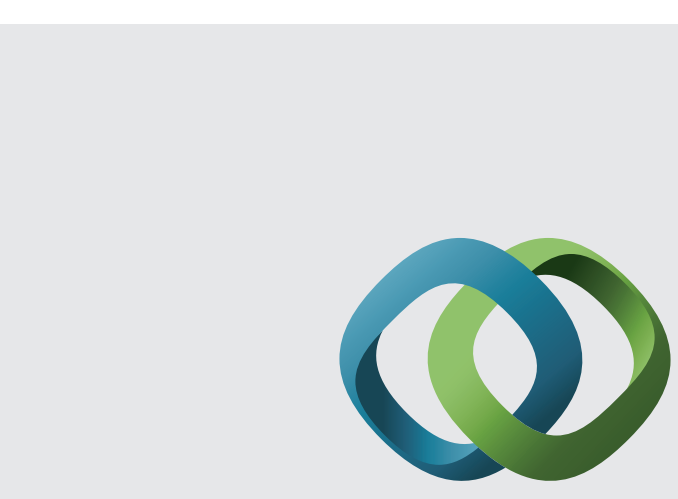

\section{Hindawi}

Submit your manuscripts at

http://www.hindawi.com
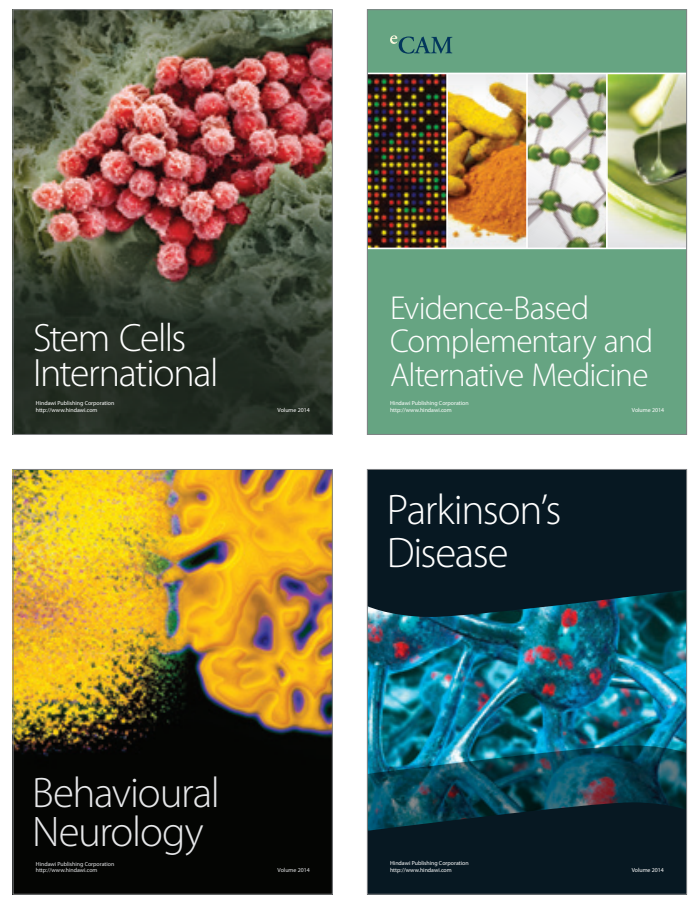
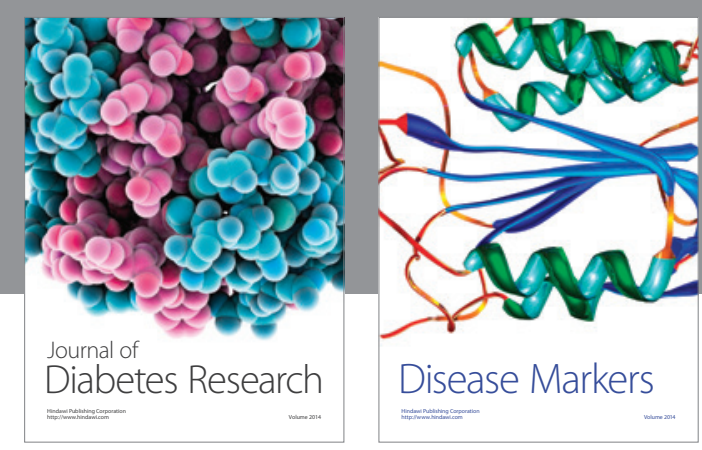

Disease Markers
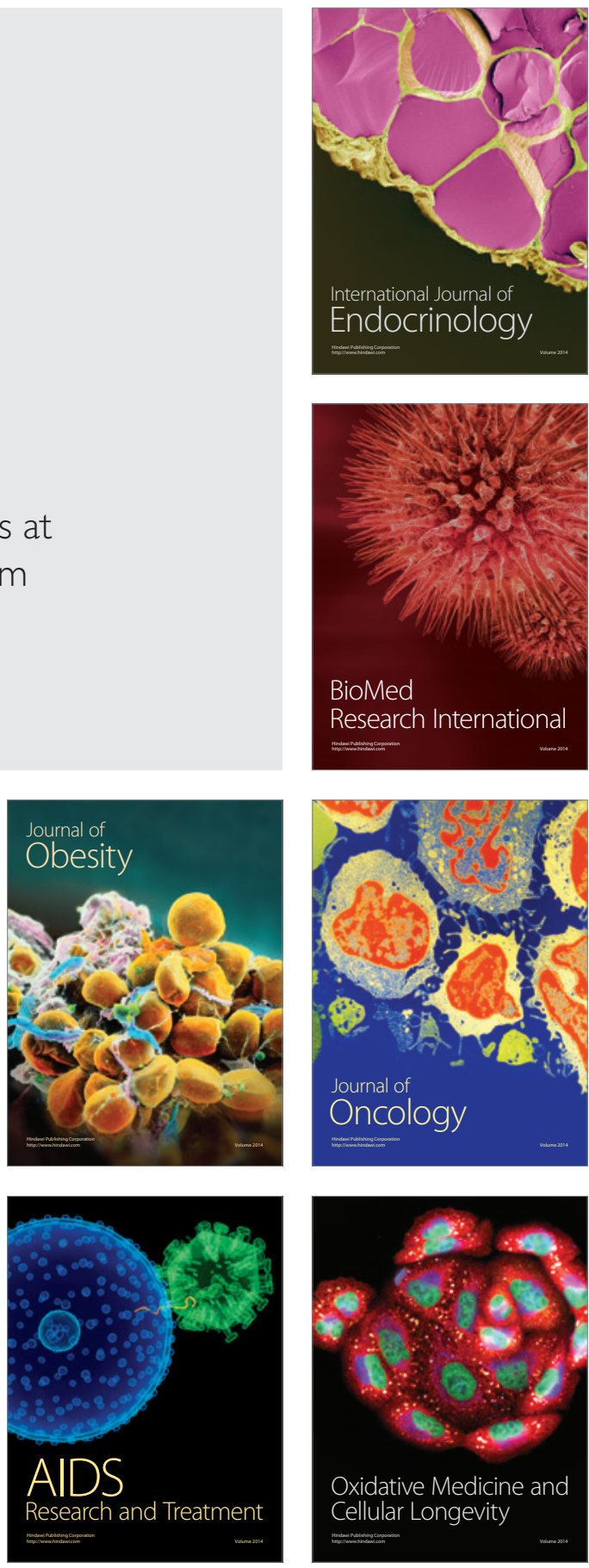